Generation and Creep of Magma in the Earth

\author{
A. C. Fowler
}

SIAM Journal on Applied Mathematics, Vol. 49, No. 1. (Feb., 1989), pp. 231-245.

Stable URL:

http://links.jstor.org/sici?sici=0036-1399\%28198902\%2949\%3A1\%3C231\%3AGACOMI\%3E2.0.CO\%3B2-R

SIAM Journal on Applied Mathematics is currently published by Society for Industrial and Applied Mathematics.

Your use of the JSTOR archive indicates your acceptance of JSTOR's Terms and Conditions of Use, available at

http://www.jstor.org/about/terms.html. JSTOR's Terms and Conditions of Use provides, in part, that unless you have obtained prior permission, you may not download an entire issue of a journal or multiple copies of articles, and you may use content in the JSTOR archive only for your personal, non-commercial use.

Please contact the publisher regarding any further use of this work. Publisher contact information may be obtained at http://www.jstor.org/journals/siam.html.

Each copy of any part of a JSTOR transmission must contain the same copyright notice that appears on the screen or printed page of such transmission.

The JSTOR Archive is a trusted digital repository providing for long-term preservation and access to leading academic journals and scholarly literature from around the world. The Archive is supported by libraries, scholarly societies, publishers, and foundations. It is an initiative of JSTOR, a not-for-profit organization with a mission to help the scholarly community take advantage of advances in technology. For more information regarding JSTOR, please contact support@jstor.org. 


\title{
GENERATION AND CREEP OF MAGMA IN THE EARTH*
}

\author{
A. C. FOWLER $\dagger$
}

\begin{abstract}
This paper describes a model representing the process of partial melting of deforming mantle rock, and the associated melt migration due to differential buoyancy. The model is a double free-boundary problem of degenerate type, and is typical of such slow, reactive two-phase flows. Prescription of boundary conditions is problematical, but in some sense the model picks its own; a realistic asymptotic solution involving rather novel boundary layer behaviour is presented, for the case of two fixed boundaries, and it is then shown how the free boundaries can be determined a posteriori. The implications for geophysical behaviour are discussed.
\end{abstract}

Key words. magma transport, reactive two-phase flow, partial melting, asthenosphere

AMS(MOS) subject classification. 86, 76S05, 76T05, 35B25

1. Introduction. The generation and transport of molten magma in the earth's mantle of crystalline rocks is a problem that has aroused considerable interest recently. Other than being interesting in its own right, understanding of this process is an important prerequisite to the description of magma chamber emplacement and volcanic eruption, as well as to a rational explanation for the chemical nature of igneous rocks. There are many interesting mathematical problems involving phase change, two-phase flow, free boundaries, and other exotic topics in the general area of volcanology and petrology, and the generation and transport of magma in the mantle is, in a sense, the grand-daddy of them all.

Solid, crystalline rock melts internally in the mantle for the following reason. The mantle of the earth (some $3000 \mathrm{~km}$ deep), though solid, is in a state of continual motion due to thermal convection-it is this motion that is manifested at the earth's surface as continental drift. As mantle rock ascends (e.g., at mid-ocean ridges, or "hot-spots" such as Iceland or Hawaii), it cools by decompression (i.e., adiabatically); the solidus temperature, however (mantle rocks are a mixture of many different minerals), decreases more rapidly with pressure (on the Clapeyron curve), with the result that the rock reaches the solidus and begins to melt at some finite depth. This situation is illustrated in Fig. 1, which also shows that a cold thermal boundary layer in the lithosphere (due to "vigorous" mantle circulation, i.e., Rayleigh number »1) has the opposite effect-it promotes refreezing.

The mathematical problem we wish to address is that described above. The "heating" is internal, rather like Joule heating, and it is natural to expect a "mush" or two-phase region, to form. In fact, thermodynamic reasons suggest that the melt formed is interconnected at all volume fractions down to zero (McKenzie [8]), although this may not be generally true (Nicolas [9]). Thus we envisage that porous melt migration can take place according to Darcy's law. Such a notion dates back to Frank [7], and was extensively studied by Turcotte and Ahern [1], [16].

More recently, this subject has been re-opened for investigation independently by several authors (Fowler [4], [5], Scott and Stevenson [13], [14], McKenzie [8], Richter and McKenzie [12]), who, rather surprisingly, all adopt essentially the same generalisation of the Turcotte-Ahern model. There are two possible motivations for the generalisation. First, Turcotte and Ahern assumed that the liquid (melt) pressure was locally

* Received by the editors May 11, 1987; accepted for publication (in revised form) February 3, 1988. This research was partially supported by U.S. Navy grant N00014-86-G-0021.

$\dagger$ Mathematical Institute, 24-29 St. Giles, Oxford OX1 3LB, United Kingdom. 


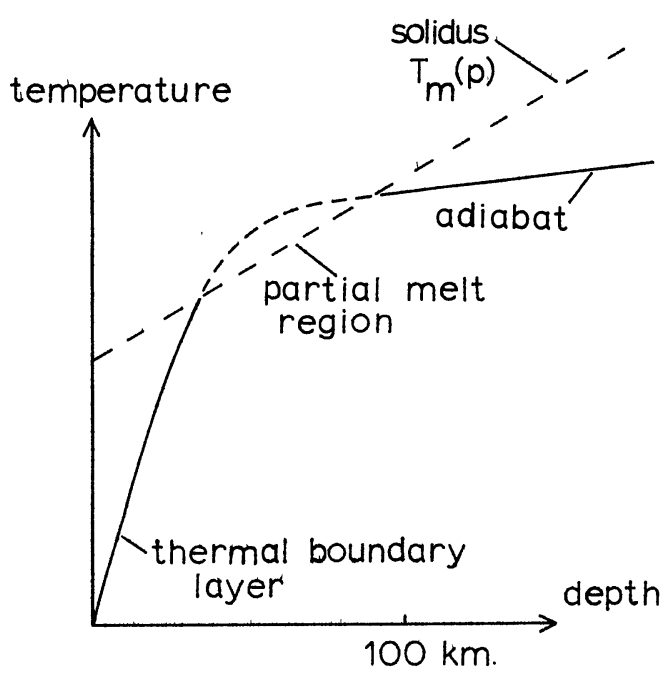

FIG. 1. Schematic illustration of the mechanism of partial melting by pressure-release. Ascending rock first begins to melt, and subsequently refreezes at the base of the lithosphere.

lithostatic; thus, melt flowed upward by differential buoyancy. In other words, $p_{l}$ (the local average liquid pressure $)=p_{s}$ (the local average solid pressure). The justification is that "crystal deformation can readily occur" so that any local differential pressure would rapidly disappear via viscous grain deformation. Turcotte and Ahern [16] state that a length scale (as mantle rock ascends) over which such deformation could occur is of order $\sim 100 \mathrm{~m}$ : very small. This "compaction length" has come to play an important part in current ideas of magma generation.

The extra complication included in theories of this decade is the explicit inclusion of a term to represent this compaction: other than that, these theories are essentially the same as (or simpler than) that of Turcotte and Ahern [16]. The relation of these various theories, and their respective merits, has been discussed by Fowler [6]. In one view (the present author's), the deformation may be represented by a constitutive law that relates liquid pressure $p_{l}$ to solid pressure $p_{s}$. The two differ by a term derived by a local microscopic model, which turns out to be, in essence, a bulk viscosity. This is quite nice, since $p_{l}$ is actually the "thermodynamic" pressure, whereas $p_{s}$ is the "dynamic pressure." We shall call this constitutive relation the compaction equation. Such microscopic modelling to derive macroscopic constitutive equations is common in two-phase flow modelling.

Given that the recent models are all essentially the same, it is surprising to what different uses they have been put. Scott and Stevenson [13], [14], Richter and McKenzie [12], and Barcilon and Richter [2] look for soliton-like solutions, which have been elegantly found in a laboratory analogue experiment (Scott et al. [15]). Arguably, these may have nothing to do with the earth. Ribe [10], [11] attempted to justify Turcotte and Ahern's quoted assumption (above) of a small compaction length. Amid all the flurry produced by these various authors, no one has actually attempted to solve the model equations put forward. The closest is Fowler [5], whose analysis is, however, wrong, as we shall show here.

Thus our aim in this paper is to study the two-phase reactive flow equations, including the compaction equation. The problem has two free (thermally determined) boundaries, but these play a small role in the dynamics of the partial melt region, 
which can be uncoupled. We show this by solving the partial melt dynamics for the case of fixed boundaries, and then show how the free boundaries may be determined subsequently. The methods are asymptotic, and we are able to analyse the solution almost completely. The novelty lies both in the weirdness of the boundary layer analysis, and in the extreme degeneracy of the boundary conditions. We seem to end up with enough, but it is not obvious; boundary conditions for this model have not been discussed by the other papers cited above.

2. Mathematical model. We consider the partially molten zone to consist of solid grains (of crystalline rock), with melt present at grain boundaries. We suppose the melt to form an interconnected network. Our variables will be locally averaged over many grains. Then the liquid velocity is given by Darcy's law

$$
\mathbf{v}=-K_{\chi} \nabla\left(p_{l}+\rho_{l} g y\right),
$$

where $\mathrm{v}$ is the melt velocity relative to the mean (barycentric) velocity, $K$ is a permeability coefficient, $\chi$ is the mass fraction of fluid, $p_{l}$ is liquid pressure, $\rho_{l}$ is liquid density, $g$ is gravity, and $y$ is the vertically upward coordinate.

Conservation of fluid mass is given by

$$
\frac{\partial}{\partial t}(\rho \chi)+\operatorname{div}[\rho \chi(\mathbf{u}+\mathbf{v})]=S,
$$

where $S$ is the rate of melting (mass per unit volume per unit time) and $\mathbf{u}$ is the barycentric velocity of the mixture. $S$ is not known a priori but is given from the energy equation since latent heat is absorbed on melting. The energy equation can be written

$$
L S+\rho c_{p} \frac{d T}{d t}=k \nabla^{2} T
$$

where $L$ is latent heat per unit mass, $c_{p}$ is the specific heat, $d / d t$ is the material derivative following the centre of mass, $k$ is the thermal conductivity, and $T$ is the temperature. In the earth's context, the obvious missing term is an adiabatic heat release, which can be shown to be moderate to small (Fowler [5]); therefore, we neglect it. Other terms have also been neglected, e.g., viscous dissipation, for similar reasons.

If we assume thermodynamic equilibrium, then $T$ is the solidus temperature and is a function of pressure and composition. As a first approach, we omit compositional effects and write

$$
T=T_{0}+\Gamma p_{l},
$$

where $\Gamma$ is the slope of the Clapeyron curve relating melting temperature and pressure. We do not assume that liquid and solid pressures are equal; rather we assume the solid grains may be differentially stressed, to which they respond by viscous creep. A microscopic model of this process leads to the (approximate) constitutive relation relating pressures,

$$
p_{s}-p_{l}=\left(\eta_{b} / \chi\right) \operatorname{div} \chi \mathbf{v}
$$

where $\eta_{b}$ is a measure of the grain viscosity, and $p_{s}$ is average solid pressure.

To complete the model, we require a prescription of $p_{s}$ and $\mathbf{u}$. Since the partially molten zone is embedded in a much larger scale flow, it is appropriate to take the pressure as approximately lithostatic:

$$
p_{s}=p_{0}-\rho_{s} g y,
$$


and to assume that the flow field $\mathbf{u}$ is kinematically prescribed. For small $\chi$, it is approximately incompressible:

$$
\operatorname{div} \mathbf{u}=0
$$

The Stefan condition can be written as

$$
\rho L \chi^{m}(\mathbf{u}+\mathbf{v}-\mathbf{V}) \cdot \mathbf{n}=\left[k \frac{\partial T}{\partial n}\right]_{c}^{m},
$$

where $m$ and $c$ denote molten and cold side, respectively. An additional restriction is implied to ensure that $T$ is below the solidus on the cold side. This will be the case if the flow is fast enough (Turcotte and Ahern [16]) and is consistent with the further boundary condition

$$
\chi=0
$$

at the lower partially molten boundary.

At either boundary, the temperature is given both by (2.4) (in the liquid) and by $T_{0}-\Gamma \sigma_{m}$ (in the solid), where $\sigma_{m}$ is the minimum compressive stress. Thus

$$
\sigma_{m}=-p_{l}
$$

at either boundary. At the upper boundary we can take $\sigma_{m} \approx-p_{s}$, whereas at the lower, this gives a condition on the mantle velocity field.

Nondimensionalisation. To nondimensionalise, we choose a typical mantle velocity scale $u_{m}$, typical depth scale $d$, time scale $d / u_{m}$, and a melt fraction scale $[\chi]$. The value of $[\chi]$ is chosen to balance the melting rate with advective heat transfer due to barycentric motion. The corresponding dimensionless equations can be written in the form

$$
\begin{gathered}
\lambda \nabla \cdot\left[\chi^{2} \nabla \varphi\right]=(\varphi+r y) \chi \\
\delta \frac{d \chi}{d t}-\nabla \cdot\left[\chi^{2} \nabla \varphi\right]+\frac{d}{d t}(\varphi-y)=\varepsilon \nabla^{2} \varphi
\end{gathered}
$$

where we have defined

$$
p_{l}+\rho_{l} g y=\rho_{l} g d \varphi+p_{0}
$$

and have used (2.1) and (2.2) as definitions to eliminate $\mathbf{v}$ and $S$. The dimensionless parameters $\lambda, \delta, \varepsilon$, and $r$ are given by

$$
\begin{aligned}
& \lambda=\eta_{b}[v] / \rho_{l} g d^{2}, \\
& \varepsilon=\kappa / u_{m} d, \\
& \delta=u_{m} /[v], \\
& r=\left(\rho_{s}-\rho_{l}\right) / \rho_{l},
\end{aligned}
$$

where the melt velocity scale $[v]$ is

$$
[v]=K \rho_{l} g[\chi],
$$

and the melt fraction scale $[\chi]$ is given by

$$
[\chi]=\left(c_{p} \Gamma u_{m} d / L K\right)^{1 / 2} .
$$


The boundary conditions we consider are the following. At the lower boundary, (2.9) applies in the same form. At the upper boundary (2.10) implies

$$
\varphi+r y=0,
$$

and the Stefan condition (2.8) becomes

$$
\chi^{m}[\mathbf{v}+\delta(\mathbf{u}-\mathbf{V})] \cdot \mathbf{n}=\varepsilon\left[\left.\frac{\partial}{\partial n}(\varphi-y)\right|_{m}-\left.\frac{\partial \theta}{\partial n}\right|_{c}\right],
$$

if we define $T=T_{0}+\Gamma p_{0}+\Gamma \rho_{l} g d \theta$ in the cold regions.

In the sequel, we shall largely be concerned with the solution of (2.11) and (2.12) between the boundaries $y_{a}$ and $y_{l}$, where, respectively, melting is initiated and refreezing occurs. In reality, these are free boundaries and must be determined by solving the temperature equation in $y<y_{a}$ and $y>y_{l}$. To see this, we define (as after (2.21))

$$
T=T_{0}+\Gamma p_{0}+\Gamma \rho_{l} g d \theta
$$

in $y<y_{a}$ and $y>y_{l}$. Then $\theta$ satisfies

$$
\frac{d \theta}{d t}=\varepsilon \nabla^{2} \theta
$$

We presume a constant temperature as $y \rightarrow-\infty$, corresponding to a vigorously convecting mantle, and by choosing the origin to be where the melting temperature at lithostatic pressure equals this far-field temperature, we can prescribe

$$
\theta \rightarrow 0 \text { as } y \rightarrow-\infty \text {. }
$$

Continuity of temperature implies

$$
\theta=\varphi-y \quad \text { at } y=y_{a} .
$$

Together with the extra Stefan condition (2.21) at $y_{a}$, this is sufficient to solve for $\theta$ in $y<y_{a}$ and determine $y_{a}$, providing $\varphi$ is known.

Above the melt zone, the diffusion equation (2.23) applies, with both (2.25) and (2.21) on $y=y_{l}$, and the prescribed surface temperature gives

$$
\theta=\theta_{0} \quad \text { at } y=y_{0}
$$

where $y_{0}$ is the dimensionless surface, and

$$
-\theta_{0}=\left(T_{m}-T_{s}\right) / \Gamma \rho_{l} g d,
$$

where $T_{m}=T_{0}+\Gamma p_{0}$ is the ambient mantle temperature and $T_{s}$ is the surface temperature. Again the extra boundary condition is sufficient to solve for both $\theta$ and $y_{l}$, providing $\varphi$ is known.

3. Analysis. The above model constitutes a free boundary value problem. In the first instance, we are less interested in the nature of the free boundary than in the structure of solutions, and whether we have enough boundary conditions. Thus, our philosophy is as follows. Assume $y_{a}$ and $y_{l}$ are fixed, and attempt to solve for $\varphi$ in $y_{a}<y<y_{l}$. This should give some insight into the behaviour of $\varphi$. Using the solution for $\varphi$, we shall then see whether $y_{a}$ and $y_{l}$ can be determined passively by solving the two temperature equations in $y<y_{a}$ and $y>y_{l}$. This will be done later in this section. Thus we suppose that the melting and freezing boundaries are pre-determined, and consequently neglect (2.21). 
The basic physics can be represented by assuming a one-dimensional solution, where now $y=0$ is the melting boundary, $y=1$ is the refreezing boundary, and $\mathbf{u}=(0,1)$ is constant. We assume a steady state. Then (2.11) and (2.12) become

$$
\begin{aligned}
& \lambda \frac{\partial}{\partial y}\left(\chi^{2} \varphi_{y}\right)=(\varphi+r y) \chi, \\
& \frac{\partial}{\partial y}\left[\delta \chi-\chi^{2} \varphi_{y}+\varphi-y\right]=\varepsilon \varphi_{y y},
\end{aligned}
$$

together with the boundary conditions (2.9) and (2.20). From (3.1), we might expect two boundary conditions for $\varphi$, and one for $\chi$; it is not entirely obvious whether (2.9) and (2.20) will be sufficient to ensure a solution: in addition, (3.1a) is degenerate when $\chi=0$. Thus it is not entirely clear what boundary conditions ought to be supplied. We shall find that explicit solution of (3.1) indicates that the boundary conditions which are naturally supplied are sufficient to determine a complete solution.

There are four parameters in the model: $\lambda, \delta, r$, and $\varepsilon$. To estimate these, we use values considered typical of mantle conditions (Fowler [5]): $\eta_{b}=10^{21}$ poise, $\rho_{s}=$ $3.5 \mathrm{gm} \mathrm{cm}^{-3}, \rho_{l}=3 \mathrm{gm} \mathrm{cm}^{-3}, g=10 \mathrm{~m} \mathrm{~s}^{-2}, d=10 \mathrm{~km}, \kappa=10^{-2} \mathrm{~cm}^{2} \mathrm{~s}^{-1}, u_{m}=1 \mathrm{~cm} y^{-1}$, $K=10^{3} \mathrm{~m}^{2} \operatorname{bar}^{-1} y^{-1}, c_{p}=.25 \mathrm{cal} \mathrm{gm}^{-1} K^{-1}, \Gamma=10^{-2} K_{\mathrm{bar}^{-1}}, L=80 \mathrm{cal} \mathrm{gm}^{-1}$. From these values, we compute

$$
\begin{aligned}
& {[\chi]=.17 \%, \quad[v]=50 \mathrm{~cm} y^{-1},} \\
& \delta \approx .02, \quad \varepsilon \approx .3, \quad \lambda \approx .5, \quad r \approx .2 .
\end{aligned}
$$

Values of $\eta_{b}$ and $u_{m}$, in particular, are liable to vary, and more reasonable may be a value of $u_{m}$ of $10 \mathrm{~cm} y^{-1}$. This changes the values above to

$$
\begin{aligned}
& {[\chi] \sim .5 \%, \quad[v] \sim 1.5 \mathrm{~m} y^{-1},} \\
& \delta \approx .06, \quad \varepsilon \approx .03, \quad \lambda \approx 1.5 .
\end{aligned}
$$

Based on these values, it seems reasonable to suppose $[\chi] \ll 1$ (as was implicitly already done), and that $\delta \sim \varepsilon \ll 1, \lambda \sim O(1)$. This assumption, at any rate, forms the basis of the subsequent analysis. The value of $\eta_{b}$ may be much lower than $10^{21}$ poise. In fact, a lower value is to be expected for values of $p_{s}-p_{l}>10$ bars (a typical mantle deviatoric stress). A much lower value of $\lambda$ (together with $\varepsilon, \delta \ll 1$ ) apparently leads to a triple singular perturbation problem of some complexity, and for the moment, we shall be content to examine the structure in the limit where $\lambda \sim O(1)$; the case $\lambda \ll 1$ is examined later.

$\delta \sim \varepsilon \ll 1, \lambda \sim 1$ : outer solution. A straightforward outer expansion of (3.1) yields, at leading order, the following problem:

$$
\begin{aligned}
& \lambda \frac{\partial}{\partial y}\left[\chi^{2} \varphi_{y}\right]=(\varphi+r y] \chi, \\
& -\frac{\partial}{\partial y}\left[\chi^{2} \varphi_{y}\right]+\varphi_{y}-1=0,
\end{aligned}
$$

a first integral of which gives

$$
\begin{aligned}
& \varphi_{y}=1+(\varphi+r y) \chi / \lambda \\
& \chi^{2} \varphi_{y}=\varphi-y-\varphi_{0},
\end{aligned}
$$


wherein $\varphi_{0}$ is a constant of integration. Thus

$$
\chi=\lambda\left(\varphi_{y}-1\right) /(\varphi+r y),
$$

so that

$$
\lambda^{2} \varphi_{y}\left(\varphi_{y}-1\right)^{2}=(\varphi+r y)^{2}\left(\varphi-y-\varphi_{0}\right) .
$$

Equation (3.7) is of first order for $\varphi$, and can be represented as

$$
\begin{aligned}
& \varphi_{y}=f(p), \\
& p=(\varphi+r y)^{2}\left(\varphi-y-\varphi_{0}\right) / \lambda^{2}, \\
& x=f(p) \Leftrightarrow p=x(x-1)^{2} .
\end{aligned}
$$

Now suppose $\varphi+r y>0$. Then (3.5a) implies $\varphi_{y}>1$, since $\chi>0$. Consequently, (3.5b) implies $\varphi>\varphi_{0}+y$. Thus the region $-r y<\varphi<\varphi_{0}+y$ is illegal. Suppose that $\varphi_{y}>1$; then (3.8) implies $p>0$, and hence $\varphi-y-\varphi_{0}>0$. Thus (from (3.5b) and (3.4b)) $\chi^{2} \varphi_{y}$ is positive and increasing. Since $\varphi+r y=0$ cannot be reached while $\varphi_{y}>1$, it follows that $\chi^{2} \varphi_{y}$ cannot reach zero (even if $\delta, \varepsilon \neq 0$ ), whence $\chi \neq 0$ and $\varphi$ cannot have a maximum. In particular, $\chi(\varphi+r y) \neq 0$ at $y=1$ for any trajectory in the region $\varphi_{y}>1$. Thus we exclude the region $\varphi+r y>0, \varphi-\varphi_{0}-y>0$ from consideration.

We now attempt to satisfy (2.9). Suppose that the outer solution satisfies $\chi=0$ at $y=0$. Then (3.5a) implies (for the outer solution) $\varphi_{y}=1$ at $y=0$. Equations (3.8) imply $p=0$, whence $\varphi=\varphi_{0}$ at $y=0$, or $\varphi=0$. If $\varphi=0$, then we go (for $y>0$ ) into the excluded region $\varphi_{y}>1$. If $\varphi(0)=\varphi_{0}$ and $\varphi_{y}=1$, then for small $y$, (3.5) implies $\varphi_{y}<1$, and $\varphi>\varphi_{0}+y$, which are contradictory. Apparently, we cannot force $\chi=0$ for the outer problem. Therefore we endeavour to find a boundary layer near $y=0$, so that $\chi$ can equal zero at the boundary.

Boundary layer at $\boldsymbol{y}=\mathbf{0}$. Let us assume $\varepsilon \sim \delta$, and specifically put

$$
\varepsilon=c \delta, \quad c \sim O(1) .
$$

We put

$$
y=\delta Y, \quad \varphi \sim \varphi^{(0)}+\delta \varphi^{(1)}+\cdots, \quad \chi \sim \chi^{(0)}+\cdots,
$$

to obtain a sequence of problems from (3.1). At $O(1)$,

$$
\frac{\partial}{\partial Y}\left[\chi^{(0) 2} \varphi_{Y}^{(0)}\right]=0, \quad \varphi_{Y}^{(0)}=c \varphi_{Y Y}^{(0)}
$$

(here we have first substituted for $\left(\chi^{2} \varphi_{y}\right)_{y}$ from (3.1a) into (3.1b)); the solution that can match to an outer solution is

$$
\varphi^{(0)}=\text { constant }
$$

At order $\delta$, we have

$$
\frac{\partial}{\partial Y}\left[\chi^{(0) 2} \varphi_{Y}^{(1)}\right]=0, \quad \chi_{Y}^{(0)}+\varphi_{Y}^{(1)}-1-\varphi^{(0)} \chi^{(0)} / \lambda=c \varphi_{Y Y}^{(1)},
$$

whence

$$
\chi^{(0) 2} \varphi_{Y}^{(1)}=\text { constant }=0,
$$

if we require $\chi^{(0)}=0$ on $Y=0$. Thus

$$
\varphi^{(1)}=\text { constant. }
$$


From (3.12) and (3.15), matching to the outer solution requires $\varphi_{y} \rightarrow 0$ as $y \rightarrow 0$ (for the outer solution); thus $p$ in $(3.8)=0$ at $y=0$, whence (since $\varphi \neq 0$ from the outer solution), $\varphi$ (outer) $\rightarrow \varphi_{0}$ as $y \rightarrow 0$. Without any loss of generality, we can take

$$
\varphi^{(0)}=\varphi_{0}, \quad \varphi^{(1)}=0 .
$$

Equation (3.13) now reduces to

$$
\chi_{Y}^{(0)}-1-\varphi_{0} \chi^{(0)} / \lambda=0
$$

whose solution satisfying $\chi^{(0)}=0$ on $Y=0$ is

$$
\chi^{(0)}=-\left(\lambda / \varphi_{0}\right)\left[1-\exp \left(\varphi_{0} Y / \lambda\right)\right] .
$$

(a)

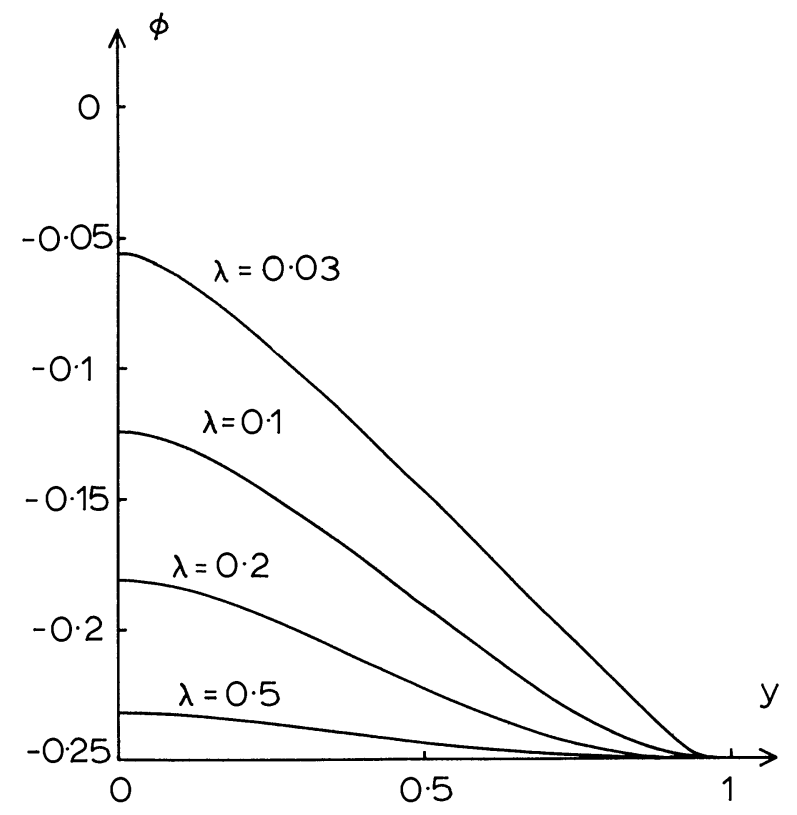

(b)

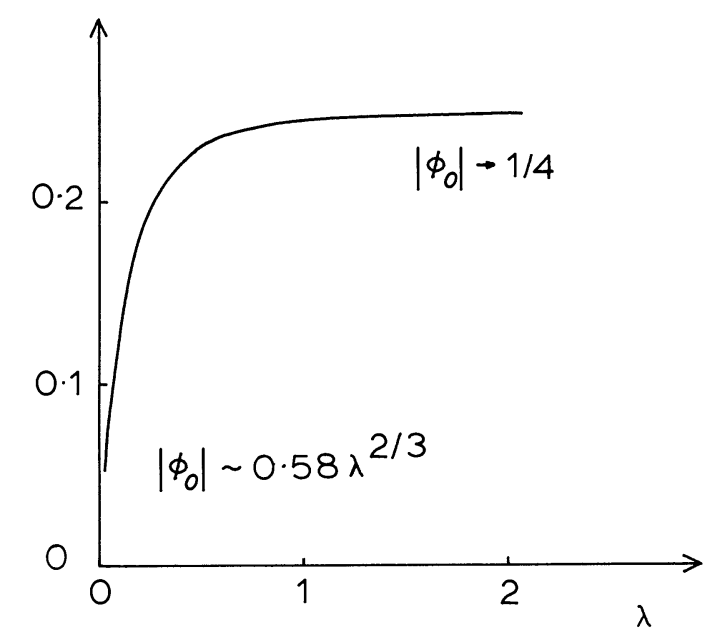

FIG. 2. (a) Solutions of (3.7) for various values of $\lambda$. (b) The variation of $\left|\varphi_{0}\right|$ with $\lambda$. (Here $r=\frac{1}{4}$.) 
Evidently, we require $\varphi_{0}<0$ for matching to be possible, and the leading order boundary conditions for the outer problem (3.5) are

$$
\varphi=\varphi_{0}, \quad \chi=\lambda /\left|\varphi_{0}\right| \quad \text { on } y=0 .
$$

Thus the outer solution has (at least, for small y) $\varphi_{y}<0, \varphi-y-\varphi_{0}<0$, as well as $\varphi+r y<0$. As long as $\varphi_{y}<0$, then $-\chi^{2} \varphi_{y}$ is positive and increasing, and this is true (from (3.5b) irrespective of the size of $\delta$ and $\varepsilon$. It follows that no boundary layer structure for $\varphi$ can enable $(\varphi+r y)=0$ to hold at $y=1$, but that satisfaction of this condition seems to necessitate that, to leading order, the outer solution to (3.5) satisfies (2.20), i.e., $\varphi+r y=0$ at $y=1$. That is, we solve the first-order equation (3.5) with $\varphi=\varphi_{0}$ on $y=0$ and $\varphi=-r$ on $y=1$. The extra condition enables $\varphi_{0}$ to be chosen. That such a problem indeed has a solution is evidenced by the numerical solutions shown in Fig. 2. It can be seen that $\varphi_{y}<0, \varphi+r y<0$. Physically, this implies $p_{s}>p_{l}$, with the effective pressure decreasing toward zero as $y$ increases.

This seems fine, until we realise that although we have followed $\varphi$ with $\varphi_{y}<0$, precise prescription of $\varphi=-r$ at $y=1$, and consequently $\varphi_{y}=0$ there (from (3.8), and since $\varphi_{y}$ varies continuously with $\varphi$ and $y$ ) requires (from (3.5a)) that $\chi \rightarrow \infty$ there. Consequently, (3.5) cannot be uniformly valid near $y=1$, and a further boundary layer structure is necessary to obtain finite $\chi$.

$$
\varphi+r \sim d(1-y)^{3}
$$

where

$$
d=r^{2}\left(1+r+\varphi_{0}\right) / 3 \lambda^{2},
$$

and $d>0$ since $\left(\varphi_{y}<0\right)-\varphi_{0}<r$. Also $\chi \sim(1-y)^{-1}$; thus we define

$$
\varphi=-r+\delta^{3 \alpha} \Phi, \quad \chi=\delta^{-\alpha} \Psi, \quad y=1-\delta^{\alpha} Z,
$$

where $\alpha$ is yet to be chosen. Matching conditions are, from (3.20) and (3.5a),

$$
\Phi \sim d Z^{3}, \quad \Psi \sim \lambda / r Z \text { as } Z \rightarrow \infty .
$$

A leading-order balance in (3.1b) is effected if $\alpha=\frac{1}{2}$; thus $1-y \sim \delta^{1 / 2}, \chi \sim \delta^{-1 / 2}$. Then (3.1) becomes, to leading order,

$$
\frac{\partial}{\partial Z}\left[\Psi^{2} \Phi_{Z}\right]=O\left(\delta^{2} / \lambda\right)
$$

and

$$
-\Psi_{Z}+r Z \Psi / \lambda-1=O\left(\varepsilon \delta^{1 / 2}, \delta\right) .
$$

Neglecting higher-order terms yields

$$
\Psi^{2} \Phi_{Z}=\text { constant }=3 d \lambda^{2} / r^{2},
$$

and

$$
\Psi_{Z}=r Z \Psi / \lambda-1, \quad \Psi \sim \lambda / r Z \text { as } Z \rightarrow \infty
$$

whose solution is

$$
\Psi=\int_{Z}^{\infty} \exp \left[\left(\frac{r}{2 \lambda}\right)\left(Z^{2}-w^{2}\right)\right] d w
$$


In particular, on $Z=0$,

$$
\Psi=\int_{0}^{\infty} \exp \left(-\frac{r w^{2}}{2 \lambda}\right) d w=\left(\frac{\lambda \pi}{2 r}\right)^{1 / 2},
$$

i.e.,

$$
\chi=(\lambda \pi / 2 r \delta)^{1 / 2} \text { on } y=1 .
$$

Thus we obtain Fig. 3. The melt fraction jumps rapidly at both top and bottom, whereas the liquid pressure varies smoothly. The driving head $\varphi$ directs melt flow upward, but the effective pressure $p_{s}-p_{l} \propto-(\varphi+r y)$ is positive and decreases toward zero as $y$ tends to one.

The free boundaries $y_{a}$ and $y_{l}$. Now we return to the free boundary nature of the upper and lower boundaries of the partial melt zone. There are two aspects. First, we choose the origin so that (2.24) is satisfied. Thus a temperature precursor will cause $y_{a}<0$. If we denote the solution obtained above by $\varphi=\varphi_{c}(y), \chi=\chi_{c}(y)$, and assume for the moment we can choose $y_{l}=1+y_{a}$, then the solution of (3.1) with $\chi=0$ on $y=y_{a}$ is just

$$
\varphi=-r y_{a}+\varphi_{c}\left(y-y_{a}\right),
$$

and in particular, $\varphi+r y \neq 0$ on $y=y_{a}$. Next we show that $y_{a}$ can be consistently found (as $O(1)$ ) from the temperature solution in $y<y_{a}$. (This was also done by Turcotte and Ahern [16].)

With the assumption

$$
\varepsilon=c \delta, \quad c \sim O(1), \quad \delta \ll 1
$$

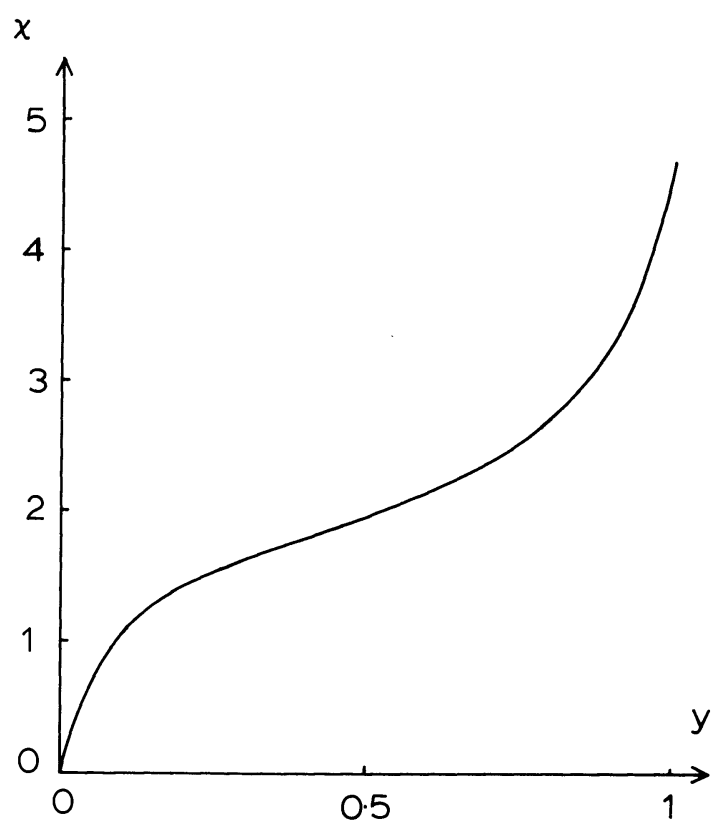

FIG. 3. Typical form of $\chi$ versus $y$, using the outer numerical solution together with the two boundary layers. The boundary layer solution at $y=1$ is approximated by $\chi \approx \lambda /\left[r\left(1-y+(2 \delta \lambda / \pi r)^{1 / 2}\right)\right]+O(\delta)$, rather than by (3.28), which is numerically inconvenient. The above expression satisfies both (3.30), and the matching condition to the outer solution. Parameter values $\delta=.06, \lambda=.2$ are used. 
and supposing $\mathbf{u}=(0,1)$ as before, we must solve

$$
\theta_{y}=\varepsilon \theta_{y y} \quad \text { in } y<y_{a}
$$

with

$$
\theta \rightarrow 0 \text { as } y \rightarrow-\infty
$$

and, from (2.21) and (2.25)

$$
\theta=-\left|\varphi_{0}\right|-(1+r) y_{a}, \quad \frac{\partial \theta}{\partial y}=-1 \quad \text { on } y_{a},
$$

where we have used $\varphi_{c}=\varphi_{0}, \varphi_{y}=0$ on $y=y_{a}$, together with (3.31). The solution is

$$
\theta=-\varepsilon \exp \left[\left(y-y_{a}\right) / \varepsilon\right], \quad y_{a}=-\left[\left|\varphi_{0}\right|-\varepsilon\right] /(1+r),
$$

so that $\theta=O(\varepsilon), y_{a}=O(1)$.

We allowed an assumption above that $y_{l}=1+y_{a}$, and this can be justified as follows. The free boundary nature of $y_{a}$ and $y_{l}$ means that in reality the depth scale $d$ is unknown in advance: our assumption of $d=10 \mathrm{~km}$ is simply a reasonable guess. Thus we can choose $y_{l}$ as we wish, provided we keep $d$ (or more generally, one of the dimensionless parameters) to be chosen. We do this by allowing $\varepsilon$ to be chosen at the end to enforce the top surface condition. That is, the procedure is to suppose $\varepsilon \sim \delta \ll 1$, $\lambda \sim 1$ in advance, and then determine $\varepsilon$ to be consistent with the extra temperature condition in $y>y_{l}$. We then hope that this value is consistent with our estimate. In fact, it is evident from our analysis that we only require $\delta, \varepsilon \ll 1$ for self-consistency. The distinguished limit $\varepsilon \sim \delta$ is not necessary.

The question of what mantle velocity field $\mathbf{u}$ we should assume arises. In reality, the lithosphere moves laterally in oceanic regions, but possibly not in continental regions. Thus a simple, realistic assumption for continental lithosphere is that $\mathbf{u}=\mathbf{0}$ for $y>y_{l}$. But in this case we cannot realistically have $\mathbf{u}=(0,1)$ in $y<y_{l}$, as before. A better choice of (incompressible) velocity field is $\mathbf{u}=\left(x, y_{l}-y\right)$. However, solutions for $\varphi$ and $\chi$ in $\left(y_{a}, y_{l}\right)$ and for $\theta$ in $y<y_{a}$ still only depend on $y$ as long as the temperature at $y=-\infty$ is spatially uniform, and we shall suppose the boundary layer structure is comparable. There may still be a certain amount of "underplating" due to melt refreezing at $y_{l}$. This is of order $[\chi][v]$, which might typically be in the range of $1-10 \mathrm{~mm} y^{-1}$ (from (3.2) and (3.3)); this is actually comparable to normal continental erosion rates, so that it could make sense to prescribe a steady state for continental lithosphere in which any underplating is balanced by erosion, and in which $\mathbf{u}=\mathbf{0}$. A conductive profile is actually also appropriate to oceanic lithosphere (e.g., under Hawaii), where rapid lateral advection ensures a high vertical heat transport.

With $\mathbf{u}=\mathbf{0}$ in $y>y_{l}$, we have $\varphi_{c}(1)=-r$, and thus, from (3.31), $\varphi=-r\left(1+y_{a}\right)$. The boundary conditions for $\theta$ in $y>y_{l}$ are thus, from (2.21) and (2.25), using also (3.26), (3.22), and (3.21),

$$
\begin{aligned}
& \theta=-(1+r)\left(1+y_{a}\right), \\
& \theta_{y}=-\left(1+r+\left|\varphi_{0}\right|\right) / \varepsilon \quad \text { on } y=y_{l}=1+y_{a},
\end{aligned}
$$

with solution (to $\theta_{y y}=0$ )

$$
\theta=-(1+r)\left(1+y_{a}\right)-\left(1+r+\left|\varphi_{0}\right|\right)\left(y-y_{l}\right) / \varepsilon,
$$

and satisfaction of (2.26) requires

$$
y_{0}-y_{l}=\varepsilon\left[\left|\theta_{0}\right|-(1+r)\left(1+y_{a}\right)\right] /\left(1+r+\left|\varphi_{0}\right|\right) .
$$


To estimate $y_{0}$ and $\left|\theta_{0}\right|$, we use a partial melting depth $d_{a}$ of $100 \mathrm{~km}$. With $d=10 \mathrm{~km}$, we might estimate

$$
y_{0}=d_{a} / d \approx 10 \text {. }
$$

For $\theta_{0}$ given by (2.27), we estimate $T_{m}-T_{s} \approx 1200 \mathrm{~K}, \rho_{l} g \Gamma \approx 3 \mathrm{~K} \mathrm{~km}^{-1}$, and thus

$$
d_{c}=\left(T_{m}-T_{s}\right) / \rho_{l} g \Gamma \approx 400 \mathrm{~km},
$$

so that

$$
\left|\theta_{0}\right|=d_{c} / d \approx 40 \text {. }
$$

So long as $d \ll d_{a}, d_{c}$, (3.39) roughly gives

$$
\varepsilon=y_{0} /\left|\theta_{0}\right|=d_{a} / d_{c},
$$

and thus our whole analysis is self-consistent, providing the depth of melting $d_{a}$ is much less than the "Clapeyron depth" $d_{c}$. With $d_{a}=100 \mathrm{~km}$, we have $\varepsilon=\frac{1}{4}$, corresponding to (3.2), and the interval of melting $d=\kappa / u_{m} \varepsilon$ is $10 \mathrm{~km}$ for $u_{m}=1 \mathrm{~cm} \mathrm{y}^{-1}$, and less for larger $u_{m}$.

The above shows, at least conceptually, how the partial melt problem may be usefully uncoupled from the free boundary location, but further consideration is necessary to give more precise information.

The case $\lambda \ll 1$. The grain closure viscosity $\eta_{b}$ in (2.14) is generally considered to depend on stress as a power law material. If $\eta_{m}$ is a local ambient viscosity at a deviatoric stress level of $\tau_{m}$, then we should properly take

$$
\eta_{b}=\eta_{m}\left[\tau_{m} /\left(p_{s}-p_{l}\right)\right]^{n-1},
$$

and thus replace $\lambda\left(=\lambda_{m}\right)$ in $(2.14)$ by $\lambda_{b} /|\varphi+r y|^{n-1}$, where

$$
\lambda_{b}=\lambda_{m}\left(\tau_{m} / \rho_{l} g d\right)^{n-1} \text {. }
$$

That is, we replace $(3.1 \mathrm{a})$ by

$$
\lambda_{b} \frac{\partial}{\partial y}\left(\chi^{2} \varphi_{y}\right)=|\varphi+r y|^{n-1}(\varphi+r y) \chi .
$$

The appropriate value of $\lambda_{b}$ may be estimated from taking $n=3, \tau_{m}=10$ bars, $\rho_{l} g d=$ 3000 bars: then, if $\lambda_{m} \sim 1$, we have $\lambda_{b} \sim 10^{-5}$. Evidently, nonlinear creep would have a drastic effect on the behaviour.

The solution in this case can be altogether simpler. We attempt to satisfy (2.9) and (2.20), i.e.,

$$
\chi(\varphi+r y)=0 \quad \text { on } y=0,1 .
$$

Equations (3.46) and (3.47) suggest a (uniform) approximation

$$
\varphi=-r y+\lambda_{b}^{1 / n} \tilde{\varphi}
$$

which yields

$$
-2 r \chi \chi \chi_{y}+\lambda_{b}^{1 / n} \frac{\partial}{\partial y}\left(\chi^{2} \tilde{\varphi}_{y}\right)=|\tilde{\varphi}|^{n-1} \tilde{\varphi} \chi
$$

and, from (3.1b)

$$
\delta \chi_{y}+2 r \chi \chi_{y}-(1+r)=\lambda_{b}^{1 / n}\left[\varepsilon \tilde{\varphi}_{y y}+\left(\chi^{2} \tilde{\varphi}_{y}\right)_{y}-\tilde{\varphi}_{y}\right],
$$

with boundary conditions

$$
\chi \tilde{\varphi}=0 \quad \text { on } y=0,1 .
$$


Based on our experience with the case $\lambda \sim O(1)$, it is now reasonable to seek a solution to this problem as $\lambda_{b}^{1 / n} \rightarrow 0$, but with $\delta \neq 0$. Neglecting $O\left(\lambda_{b}^{1 / n}\right)$ in (3.49) and (3.50), we find that $\chi$ is given by

$$
\delta \chi+r \chi^{2}=(1+r) y
$$

so that $\tilde{\varphi}(<0)$ is given from (3.49) by

$$
(-\tilde{\varphi})^{n}=2 r \chi_{y}=2 r(1+r) /(\delta+2 r \chi) .
$$

Thus

$$
\tilde{\varphi} \sim-[r(1+r) / y]^{n / 2}, \quad y \sim O(1),
$$

but when $y \sim \delta^{2}$, then $\chi \sim \delta$, and $\tilde{\varphi} \sim \delta^{-1 / n}$. In particular, $\tilde{\varphi}_{0}=\tilde{\varphi}$ at $y=0$ is given by

$$
\tilde{\varphi}_{0}=-[2 r(1+r) / \delta]^{1 / n} \text {. }
$$

Thus there is a boundary layer of dimensional length $\delta^{2} d=L u_{m} / \kappa \rho_{l}^{2} g^{2} c_{p} \Gamma$, a compaction zone, over which $p_{s}-p_{l}$ drops from $O\left[\rho_{l} g d\left(\lambda_{b} / \delta\right)^{1 / n}\right]$ to a value smaller by a factor $O\left(\delta^{1 / n}\right)$. However, this solution is only found to be valid if $\lambda_{b}^{1 / n} \ll \delta^{2+(1 / n)}$, an unlikely circumstance. If we suppose on the other hand that $\lambda_{b}^{1 / n} \gg \delta^{2+(1 / n)}$, then we find that there is a boundary layer of thickness $\lambda_{b}^{2 /(2 n+1)}$, corresponding to a dimensionless thickness of

$$
\delta_{c}=\lambda_{b}^{2 /(2 n+1)} d=\left[\eta_{b}^{2}\left(\tau_{m} / \rho_{l} g\right)^{2(n-1)}\left(c_{p} \Gamma u_{m} K / L\right)\right]^{1 /(2 n+1)},
$$

wherein the effective pressure $p_{s}-p_{l}$ drops from $O\left[\rho_{l} g d \lambda_{b}^{2 /(2 n+1)}\right]$ to a value $O\left[\rho_{l} g d \lambda_{b}^{1 / n}\right]$. A typical thickness of this compaction zone, with $\lambda_{b}=10^{-5}, d=10 \mathrm{~km}$, is $400 \mathrm{~m}$, and a typical pressure excess is $\sim 100$ bars. Beyond it, the pressure excess is a little lower, but not zero, and perhaps of order 60 bars. The equations satisfied in this zone are just those for the outer problem (3.4) (with $n \neq 1$ ), and thus we deduce that within the compaction zone there is a further boundary layer in which $\chi$ jumps, and that the previous analysis for $\lambda \sim O(1)$ carries across uniformly providing $\lambda_{b} \gg \delta^{2 n+1}$. In particular, we disagree with the implied results of other authors that the effective pressure tends to zero beyond the compaction zone (McKenzie [8], Ribe [10], Ahern and Turcotte [1]).

4. Conclusions. We have presented and analysed a model for the porous transport of magma through partially molten rock. Various conclusions may be drawn, and various questions can be raised as to the validity of these.

The principal conclusions we wish to draw are these. There is an effective pressure $\left(p_{s}-p_{l}\right)$ in the partially molten zone, typically of the order of $60-100$ bars, with the higher values prevailing in a "compaction zone" of some $400 \mathrm{~m}$ thickness at the base of the zone. Toward the top of the zone, the effective stress tends to zero, which will facilitate fracture of the molten rock. We then expect that such fractures can migrate into the lithosphere, as described by Emerman et al. [3].

The model from which these conclusions are drawn represents a reactive porous medium, subject to compaction and interphase melting. The model is to some extent speculative, particularly in how the compaction process is modelled, but several recent independent efforts in this direction have all derived the same form for the governing equations (McKenzie [8], Scott and Stevenson [14], Fowler [5]), so that some consensus is possible.

However, we can take different quantitative forms for, in particular, the porosity and the compaction (bulk) viscosity, so that the precise quantitative results may be debated. It is difficult to compare the present analysis with that of other authors, since 
the present analysis is the only one that consistently aims to solve the governing equations in their entirety. Other analyses (particularly those concerning "magmons," or travelling wave solutions; Scott and Stevenson [13], [14], Richter and McKenzie [12], Barcilon and Richter [2]) exclude melting (but include compaction) by, for example, ignoring (2.3) (the energy equation) and setting $S=0$ in (2.2). This is an arbitrary and, we would argue, an unjustifiable approach.

The mathematical model we obtain is a complicated double free boundary one. Nevertheless, we are able to split the problem into two parts, since the dynamics of partial melt migration appears to uncouple from the determination of the free boundaries, which can then be found subsequently in a straightforward manner. The equations governing the partial melt dynamics are of third order (see (2.11) and (2.12)), and we should expect three boundary conditions, one for $\chi$ and the others for $\varphi$. However, because of the degeneracy, it is not entirely evident that three are indeed necessary. In fact, we find that prescription of two $(\chi=0$ at $y=0$ and $\varphi+r y=0$ at $y=1)$ are sufficient to determine the solution. The first of these is an "entropy jump" condition to ensure that the subzone solid rock is not superheated, while the second is a thermodynamic equilibrium assumption.

The model we analyse is curious because of its double boundary layer structure. It has one degenerate second derivative for $\varphi$ (the term $\left.\left(\chi^{2} \varphi_{y}\right)_{y}\right)$, one singularly perturbed second derivative $\left.\left(\varepsilon \varphi_{y y}\right)\right)$, and one singularly perturbed first derivative for $\chi\left(\delta \chi_{y}\right)$. And yet the term $\varepsilon \varphi_{y y}$ is actually regular, and both boundary layers involve rapid changes in $\chi$-despite the fact that, on the face of it, $\varphi$ should increase exponentially out of that at $y=0$. We shudder to think what difficulties a straightforward computational approach would encounter.

Acknowledgments. I am indebted to Paul Wilmott, with whom the structure of the solution given in $\S 3$ was worked out. I thank David Stevenson and David Scott for keeping me up to date on their work on this problem.

\section{REFERENCES}

[1] J. L. Ahern AND D. L. TurcotTe, Magma migration beneath an ocean ridge, Earth Planet. Sci. Lett., 45 (1979), pp. 115-122.

[2] V. BARCilon AND F. M. Richter, Nonlinear waves in compacting media, J. Fluid Mech., 164 (1986), pp. 429-448.

[3] S. H. Emerman, D. L. Turcotte, And D. A. Spence, Transport of magma and hydrothermal solutions by laminar and turbulent fluid fracture, Phys. Earth Planet Int., 41 (1986), pp. 249-259.

[4] A. C. FOWLER, On the transport of moisture in polythermal glaciers, Geophys. Astrophys. Fluid Dynamics, 29 (1984), pp. 99-140.

[5] — A mathematical model of magma transport in the asthenosphere, Geophys. Astrophys. Fluid Dynamics, 33 (1985), pp. 63-96.

[6] - Theories of mushy zones: applications to alloy solidification, magma transport, frost heave and igneous intrusions, in Structure and Dynamics of Partially Solidified Systems, D. Loper, ed., NATO ASI series, Martinus Nijhoff, Dordrecht, 1987, pp. 161-199.

[7] F. C. FRANK, Two-component flow model for convection in the earth's upper mantle, Nature, 220 (1968), pp. 350-352.

[8] D. P. MCKenZie, The generation and compaction of partial melts, J. Petrol., 25 (1984), pp. 713-765.

[9] A. Nicolas, A melt extraction model based on structural studies in mantle peridotites, J. Petrol., 27 (1986), pp. 999-1022.

[10] N. M. RiBE, The deformation and compaction of partial molten zones, Geophys. J. R. Astr. Soc., 83 (1985), pp. 487-501.

[11] - The generation and composition of partial melts in the earth's mantle, Earth Planet. Sci. Letts., 73 (1985), pp. 361-376. 
[12] F. M. Richter AND D. P. MCKenzie, Dynamical models for melt segregation from a deformable matrix, J. Geol., 92 (1984), pp. 729-740.

[13] D. R. Scott And D. J. Stevenson, Magma solitons, Geophys. Res. Letts., 11 (1984), pp. 1161-1164.

[14] — Magma ascent by porous flow, J. Geophys. Res., 91 (1986), pp. 9283-9296.

[15] D. R. Scott, D. J. Stevenson, AND J. A. WhiteheAd, JR., Observations of solitary waves in a viscously deformable pipe, Nature, 319 (1986), pp. 759-761.

[16] D. L. Turcotte And J. L. Ahern, A porous flow model for magma migration in the asthenosphere, J. Geophys. Res., 83 (1983), pp. 767-772. 